\title{
Ras pathway activation in gliomas: a strategic target for intranasal administration of perillyl alcohol
}

\author{
Clovis Orlando da Fonseca ${ }^{1}$, Rafael Linden ${ }^{2}$, Débora Futuro ${ }^{3}$, Cerli Rocha Gattass ${ }^{4}$ \\ and Thereza Quirico-Santos ${ }^{4,5}$
}

${ }^{1}$ Serviço de Neurocirurgia, Hospital Universitário Antônio Pedro, Universidade Federal Fluminense, Niterói 24030-210, RJ, Brazil

${ }^{2}$ Laboratório de Neurogênese, Universidade Federal do Rio de Janeiro, Rio de Janeiro, R.J, Brazil

3 Faculdade de Farmácia, Universidade Federal Fluminense, Niterói 24030-210, RJ, Brazil

${ }^{4}$ Laboratorio de Imunologia Celular, Universidade Federal do Rio de Janeiro, 21949-900 Rio de Janeiro, RJ, Brazil

5 Departamento de Biologia Celular e Molecular, Instituto de Biologia, Universidade Federal Fluminense, 24020-150 Niterói, RJ, Brazil

Received: 2008.02.04, Accepted: 2008.06.28, Published online first: 2008.07.29

\begin{abstract}
Introduction: Targeted therapy directed at specific molecular alterations is already creating a shift in the treatment of cancer patients. Malignant gliomas commonly overexpress the oncogenes EGFR and PDGFR and contain mutations and deletions of the tumor suppressor genes PTEN and TP53. Some of these alterations lead to activation of the P13K/Akt and Ras/MAPK pathways, which provide targets for therapy. Perillyl alcohol $(\mathrm{POH})$, the isoprenoid of greatest clinical interest, was initially considered to inhibit farnesyl protein transferase. Follow-up studies revealed that POH suppresses the synthesis of small $\mathrm{G}$ proteins, including Ras. Intranasal delivery allows drugs that do not cross the blood-brain barrier to enter the central nervous system. Moreover, it eliminates the need for systemic delivery, thereby reducing unwanted systemic side effects.

Materials and Methods: Applying this method, a phase I/II clinical trial of POH was performed in patients with relapsed malignant gliomas after standard treatment: surgery, radiotherapy, and chemotherapy. $\mathrm{POH}$ was administrated in a concentration of $0.3 \%$ volume/volume $(55 \mathrm{mg})$ four times daily in an interrupted administration schedule. The objective was to evaluate toxicity and progression-free survival (PFS) after six months of treatment. The cohort consisted of 37 patients, including 29 with glioblastoma multiforme (GBM), 5 with grade III astrocytoma (AA), and 3 with anaplastic oligodendroglioma (AO). Neurological examination and suitable image analysis (computed tomography (CT), magnetic resonance imaging (MRI)) established disease progression. Complete response was defined as neurological stability or improvement of conditions, disappearance of CT/MRI tumor image, and corticosteroid withdraw; partial response (PR) as $\geq 50 \%$ reduction of $\mathrm{CT} / \mathrm{MRI}$ tumor image, neurological stability, or improvement of conditions and corticosteroid requirement; progressive course (PC) as $\geq 25 \%$ increase in CT/MRI tumor image or the appearance of a new lesion; and stable disease as a lack of any changes in the $\mathrm{CT} / \mathrm{MR}$ tumor image or neurological status.

Results: After six months of treatment, PR was observed in $3.4 \%(\mathrm{n}=1)$ of the patients with GBM and $33.3 \%(\mathrm{n}=1)$ with AO; stable disease in $44.8 \%(n=13)$ with GBM, $60 \%(n=3)$ with AA, and $33.3 \%(n=1)$ with AO; and PC in $51.7 \%(n=15)$ with GBM, $40 \%(n=2)$, with AA and $33.3 \%(n=1)$ AO. PFS (sum of PRs and stable disease) was $48.2 \%$ for GBM, $60 \%$ for $\mathrm{AA}$, and $66.6 \%$ for $\mathrm{AO}$ patients.

Conclusions: The preliminary results indicate that intranasal administration of the signal transduction inhibitor POH is a safe, noninvasive, and low-cost method. There were no toxicity events and the regression of tumor size in some patients is suggestive of antitumor activity.
\end{abstract}

Key words: perillyl alcohol, intranasal administration, gliomas, Ras.

Corresponding author: Clovis Orlando da Fonseca, Serviço de Neurocirurgia, Hospital Universitário Antônio Pedro, Departamento de Cirurgia Geral e Especializada, Centro de Ciências Médicas, CEP 24030-210, Niterói, RJ, Brazil, e-mail: clovis.orlando@uol.com.br 


\section{INTRODUCTION}

Gliomas form a heterogeneous group of tumors. In adult patients, most of these can be classified based on their histopathological features as astrocytic, oligodendroglial, oligoastrocytic, or ependymal tumors [6]. Additionally, a malignancy grade is attributed to these gliomas based on histopathological features including nuclear atypia, mitotic activity, microvascular proliferation, and necrosis. Although histopathological classification is still the gold standard guiding therapy of glioma patients, it is increasingly clear that different genetic subtypes exist within these subgroups and that specific molecular genetic changes are of prognostic significance. Molecular analysis revealed that genetic alterations and pathways may differ between the different histopathological types: whereas a gain of chromosome $7 \mathrm{p}$ and a loss of chromosome 10q are considered to be characteristic for glioblastoma multiforme (GBM; WHO grade IV astrocytoma - AA) [18], losses of $1 p$ and $19 q$ are most frequently detected in oligodendrogliomas [20]. In addition it was shown that different genetic subtypes exist even within the specific histopathological groups: secondary GBMs that develop via progression from a less malignant lesion frequently show p53 mutations, LOH $17 \mathrm{p}$, and overexpression of PDGFR, while primary GBMs that occur without clinical evidence of a precursor lesion are often characterized by amplification or overexpression of EGFR [13].

For the above reasons, research efforts have focused on the investigation of molecular alterations in neoplastic glia, aiming at the identification of novel and more specific therapeutic targets. Such alterations often result from multiple mutations in proto-oncogenes and tumor-suppressor genes [25], which encode for proteins that play important roles in signal transduction. In this context, recent discoveries have shed light on the molecular events driving glioma growth, including abnormalities of three major molecular pathways: extracellular growth factors and their receptors EGF/EGFR and PDGF/PDGFR and the signal transduction cascades Ras and AKT [21]. Therapies targeting these specific molecules may result in killing tumor cells effectively while keeping normal cells intact.

\section{The Ras pathway as a target for glioma therapy}

Ras is an integral signaling element and has been characterized as the primary switch that transmits external signals through numerous intracellular signaling pathways. In mammalian cells, three ras genes encode four Ras isoforms (H-Ras, K-Ras4A, K-Ras4B, and N-Ras) that are highly homologous but functionally distinct. Ras proteins are a class of nucleotide-binding proteins that play pivotal roles in the control of normal and transformed cell growth [26]. Experimental studies on Ras protein structure, function, and regulation indicate that they are key intermediates in signal transduction pathways that mediate proliferative and other types of signal largely upstream from receptor kinases, which control a wide variety of cellular processes including growth, differentiation, apoptosis, cytoskeletal organization, and membrane trafficking [28]. For Ras to transduce the extracellular signals provided by growth factors and cytokines, it must be associated with the inner surface of the plasma membrane. Ras proteins are a family of membrane-associated small GTPases that transmit signals from cell surface receptors such as EGFR, EGFRvIII, and PDGFR, promoting diverse cellular effects such as proliferation, survival, and angiogenesis [30]. The membrane anchorage of Ras, required for functional activity in signal transduction, is facilitated by post-translational modifications resulting in covalent attachment of a farnesyl group to the cysteine in the $\mathrm{C}$ -terminal CAAX motif. This attachment is mediated by farnesyltransferase (FTase). These lipophilic modifications facilitate the association of isoprenylated proteins with an intracellular membrane, which is a functional requirement. Farnesylation of Ras enhances its ability to stimulate downstream signaling enzymes, including mitogen-activated protein kinase in mammalian cells. Unfarnesylated Ras proteins do not associate the plasma membrane and are incapable of cellular transformation [6].

FTase inhibitors represent a new class of agents that target signal transduction pathways responsible for the proliferation and survival of diverse malignant cell types. Although these agents were developed to prevent a processing step necessary for membrane attachment and maturation of Ras proteins, recent studies suggest that FTase inhibitors block the farnesylation of additional cellular polypeptides, thereby exerting antitumor effects independent of the presence of activating ras gene mutations [21]. Even though GBMs do not display ras mutations, they may have enhanced expression of Ras. Also, GBMs expressed high levels of ligand-dependent and -independent growth factor (EGF and PDGF) receptors. Activation of these receptors leads to tyrosine kinases activation and functional up-regulation of the Ras signaling pathway or expresses the activated form of this protein [20]. Overexpression and activation of receptor tyrosine kinases, such as PDGFR and EGFR, lead to the proliferation of human malignant AA cells. Although oncogenic mutations affecting Ras are not prevalent in human malignant AAs, they might be elevated in these tumors secondary to the mitogenic signals originating from activated receptor tyrosine kinases [20]. Furthermore, blocking Ras activation by expression of the Ha-Ras-Asn dominant-negative mutant or by farnesyl transferase inhibitors decreased the in vitro proliferation of the human AA cell lines [14]. These results support the hypothesis that proliferative signals from receptor tyrosine kinases expressed by human malignant AA cells utilized the Ras mitogenic pathway [14]. Pharmacological inhibitors of the Ras pathway may therefore be of therapeutic value in these currently terminal tumors. Previous studies have demonstrated that AAs express elevated levels of activated Ras.GTP 
despite the absence of activating ras mutations [11]. Overall, the current data suggest that activation of the Ras signaling pathway in malignant gliomas is due to aberrant expression and overactivity of membrane tyrosine kinase receptors, including EGFR, PDGFR, FGFR, and IGF-IR.

\section{Perillyl alcohol-inhibition of the Ras/MAPK pathway}

The search for new chemotherapeutic drugs has increased, especially for those that have a natural origin. $\mathrm{POH}$, also known as p-metha 1,7-diene-6-ol and sometimes as 4-isopropenyl-cyclohexenecarbinol, is composed of two isoprene units produced by the mevalonate pathway. Diverse mevalonate-derived products of secondary metabolic pathways present in plants have both chemotherapeutic and chemopreventive properties. The mevalonate pathway produces isoprenoids that are vital for diverse cellular functions, ranging from cholesterol synthesis to growth control. Several mechanisms for the feedback regulation of low-density-lipoprotein receptors and of two enzymes involved in mevalonate biosynthesis ensure the production of sufficient mevalonate for several end products [5]. Manipulation of this regulatory system could be useful in treating certain forms of cancer [10]. It has been found to be active in inducing apoptosis in tumor cells with no impact on normal cells and can in fact turn back tumor cells to a differentiated state [31].

Although the mechanism by which $\mathrm{POH}$ exerts its anti-cancer activity is not clear, a number of potentially important drug-related activities have been observed in preclinical studies, including cellular effects such as an early G1 arrest and the induction of apoptosis and biochemical effects such as the inhibition of post-translational modification of proteins involved in signal transduction. Indeed it has been postulated that the anti-carcinoma activity of $\mathrm{POH}$ involves a decrease in the levels of isoprenylated Ras and Ras-related proteins, thereby reducing the physiological functioning of these proteins [16]. Protein isoprenylation involves the post-translational modification of a protein by the covalent attachment of a lipophilic farnesyl isoprenoid group to a Cys residue at or near the carboxyl terminus. Isoprenoid substrates for prenylprotein transferase enzymes include farnesyl pyrophosphate plus geranylgeranyl pyrophosphate, two intermediates in the mevalonate pathway [1]. This action was widely attributed to the inhibition of farnesyl protein transferase activity. Farnesylation is the most critical part of the process that leads to the activation of Ras, and farnesyl transferase inhibitors exert their antitumor effect in part by inhibiting Ras-mediated signaling [22]. A study showed that on H-Ras vs. K-Ras farnesylation was inhibited by perillyl alcohol (POH) [30]. Also, follow-up studies revealed that $\mathrm{POH}$ suppresses the synthesis of small $\mathrm{G}$ proteins and 3-hydroxy-3-methylglutaryl coenzyme A reductase [17].

Our experiments [7] showed that in vitro treatment with $\mathrm{POH}$ consistently inhibited proliferation, produced marked changes in cell morphology, inhibited protein synthesis, and caused marked alteration in membrane permeability and drastic changes in the cytoarchitecture of C6, U87MG, and A172 cells. We previously showed that with in vivo treatment of glioblastoma cells, $\mathrm{POH}$ showed inhibition of cell migration and anti-metastatic activity in the model of the chick embryo with the C6 cell line [31]. Such results indicate the chemotherapeutic action of $\mathrm{POH}$ by promoting cytotoxicity and arresting migration of murine and human glioblastoma cell lines. Also, another study of our group [12] showed that treatment of both human primary cultures and established cell lines of GBM (U87 and A172) with $1 \mathrm{mM}$ of $\mathrm{POH}$ led to marked alterations in cell morphology, decreased cell viability, and death by apoptosis. Given the limited toxicity of $\mathrm{POH}$ in humans, its proven efficacy in several animal models, and its potential to inhibit Ha-ras farnesylation, we conducted a new method for $\mathrm{POH}$ administration, i.e. $\mathrm{POH}$ inhalation. This intranasal administration allows $\mathrm{POH}$ to cross the bloodbrain barrier (BBB) and reach the central nervous system (CNS), eliminating the need for systemic delivery and reducing the side effects.

Based on the favorable therapeutic ratio observed with in vitro and in vivo treatment, commercial availability, low cost, and low toxicity, we developed a phase I/II clinical trial that delivers $\mathrm{POH}$ by inhalation to patients with relapsed malignant gliomas. This trial was approved by the Brazilian Committee of Ethics and Research (CONEP 9681 no. 25000.009267/2004-25, July 12, 2004).

\section{MATERIALS AND METHODS}

This study was approved by the Ethics Council of Hospital Universitario Antonio Pedro-Universidade Federal Fluminense (UFF). Informed consent was obtained from either the patient or next of kin.

\section{Patient selection}

To be selected, patients had to have histopathological diagnosis of malignant glioma. This was done during the first surgery. Thirty-seven patients with recurrent malignant gliomas were enrolled in this trial. No effective standard therapy was available for any patient who gave informed written consent for the $\mathrm{POH}$ protocol, according to CONEP 9681 no. 25000.009267/2004-25 approved on July 12, 2004. Patients with relapsed malignant gliomas, aged older than 18 years, had measurable contrast-enhancing tumor on magnetic resonance imaging (MRI), Karnofsky performance scale of $70 \%$ or higher, adequate bone marrow function, white blood cell count of $3000 / \mu$ l or higher, absolute neutrophil count of $1500 / \mu 1$ or higher, platelet count of $100,000 / \mu \mathrm{l}$ or higher, hemoglobin of $8.0 \mathrm{~g} / \mathrm{dl}$ or higher, bilirubin of $0.3 \mathrm{mg} / \mathrm{dl}$ or higher, serum glutamic oxaloacetic transaminase not more than twice the upper 
normal limit (ULN), alkaline phosphatase not more than twice the ULN, and serum creatinine of $1.6 \mathrm{mg} / \mathrm{dl}$ or less as well as stable heart rhythm, no unstable angina, no clinical evidence of congestive heart failure, no radiation therapy or chemotherapy within 4 weeks of initiation of therapy, and no mitomycin or nitrosoureas within six weeks. Pregnant women, patients with hematological malignancy, and patients taking antiepileptic drugs known to be metabolized by the cytochrome P-450 system were excluded. Patients were not allowed to take cholesterol-lowering agents during the study.

\section{Drug formulation}

POH was supplied by Hospital Universitário Antonio Pedro-UFF and formulated for inhalation by the Pharmacia Universitária-UFF according to the BR Application Number 0107262-5 December 17, 2001.

\section{Drug administration and dose escalation}

Our previous study showed anti-metastatic activity of $\mathrm{POH}$ when $\mathrm{C} 6$ murine glial cells were exposed to concentrations of $0.3-0.03 \% \mathrm{POH}$ [31]. Applying this concentration, our group studied $\mathrm{POH}$ intranasal administration in C57BL6 mice. This study showed that POH intranasal administration increased the lymphoid population in the broncoalveolar lavage and activated some of innate immunity, suggesting a possible role for $\mathrm{POH}$ as a chemotherapeutic drug also in pathological processes affecting the lung [29]. In our clinical trial all the patients received $0.3 \% \mathrm{v} / \mathrm{v} \mathrm{POH}(55 \mathrm{mg})$ four times daily by intranasal delivery, totaling $220 \mathrm{mg}$ /day. Owing to the efficacy of the intranasal delivery used in our trial, we decided to use $0.3 \%$ POH $55 \mathrm{mg}$ four times daily as the first dose escalation.

\section{Outcome}

Complete response was defined as neurological stability or improvement of conditions, disappearance of computed tomography (CT)/MRI tumor image, and corticosteroid withdraw; partial response (PR) as $\geq 50 \%$ reduction of CT/MRI tumor image, neurological stability, or improvement of conditions and corticosteroid requirement; progressive course as $\geq 25 \%$ increase in CT/MRI tumor image or the appearance of a new lesion; and stable disease as a lack of any changes in the CT/MRI tumor image or neurological status.

\section{RESULTS}

\section{Patient characteristics}

Thirty-seven patients with recurrent malignant gliomas were enrolled, including 29 patients with GBM, 5 patients with grade III AA, and 3 patients with anaplastic oligodendroglioma (AO). The ages of the
Table 1. Patients' characteristics

\begin{tabular}{lccc}
\hline & $\begin{array}{c}\text { GBM } \\
(\mathrm{n}=29)\end{array}$ & $\begin{array}{c}\text { AA } \\
(\mathrm{n}=5)\end{array}$ & $\begin{array}{c}\text { AO } \\
(\mathrm{n}=3)\end{array}$ \\
\hline $\begin{array}{l}\text { Age, years } \\
\text { median } \\
\text { range }\end{array}$ & 50 & 52.5 & 52 \\
$\begin{array}{l}\text { Sex } \\
\text { male }\end{array}$ & $38-62$ & $41-64$ & $35-69$ \\
$\quad$ female & 19 & 3 & 1 \\
Prior treatment regimens & 10 & 2 & 2 \\
$\quad$ prior radiotherapy & 29 & 5 & 3 \\
prior chemotherapy agents & 29 & 5 & 3 \\
$\quad$ anti-epileptic drug & 29 & 5 & 3 \\
$\quad$ prior corticosteroid intake & 29 & 5 & 3 \\
KPS & & & \\
$\quad 100$ & 0 & 0 & 0 \\
$\quad 90$ & 14 & 3 & 2 \\
80 & 13 & 1 & 1 \\
70 & 2 & 1 & 0 \\
\hline
\end{tabular}

Abbreviations: Anti-epileptic drug - phenytoin, phenobarbital, carbamazepine; GBM - glioblastoma multiforme; AA - anaplastic astrocytoma; AO - anaplastic oligodendroglioma; KPS - Karnofsky performance status.

patients with GBM ranged from 38-62 (median: 50), with AA from 41-64 (median: 52.5), and with AO from 35-69 (median: 52) years. Before entering the clinical trial, all the patients received prior conventional therapy (surgery, chemotherapy, and radiotherapy), were out of therapeutic possibilities, and presented recurrence (Table 1).

\section{Outcome}

With a medium follow-up of 48 weeks, the six-month progression-free survival (PFS; partial responses and stable disease) rate for the patients with GBM was 48.2\% patients (Table 2 and Fig. 1). Patients with AA and AO had a significantly better rate than the patients with GBM (Fig. 1). Time to progression and overall survival were measured from treatment initiation and analyzed by the Kaplan-Meier method including 95\% confidence intervals (Fig. 2).

In the 29 patients with GBM, 13 showed stable disease and 15 showed PD (Fig. 3 and Table 2). All patients were were receiving corticosteroids $4 \mathrm{mg} / 6$ $\mathrm{h}$ orally before entering the clinical trial. The corticosteroid was maintained during $\mathrm{POH}$ treatment in all patients. The dose of corticosteroid was decreased in the patients who presented improvement of clinical status. One patient died decurrently of septic fever, three patients of pneumonia, and one patient of thromboembolism. In one patient with recurrent GBM after six months of treatment with $\mathrm{POH}$ and steroid therapy presented fever and seizures. CT scan revealed a brain abscess. The patient underwent a right temporal-parietal craniotomy for removal of both the abscess and the rest of the recurrent tumor. The anatomopathological examinations are show in Fig. 4. Tumor samples obtained from the first and second surgical procedures 
Table 2. Parameters of efficacy in six months of POH treatment in patients with malignant gliomas

\begin{tabular}{lccc}
\hline & $\begin{array}{c}\text { GBM } \\
(\mathrm{n}=29)\end{array}$ & $\begin{array}{c}\mathrm{AA} \\
(\mathrm{n}=5)\end{array}$ & $\begin{array}{c}\mathrm{AO} \\
(\mathrm{n}=3)\end{array}$ \\
\hline Follow-up, weeks & 48 & 48 & 56 \\
\hline $\begin{array}{l}\text { Six-month progression-free survival } \\
\text { Radiographic responses }\end{array}$ & $48.2 \%$ & $60 \%$ & $66.6 \%$ \\
$\quad$ complete responses & 0 & 0 & 0 \\
$\quad$ partial responses & 1 & 0 & 1 \\
$\quad$ stable disease & 13 & 3 & 1 \\
$\quad$ progressive disease & 15 & 2 & 1 \\
Patients w/no corticosteroid intake & & & \\
$\quad \begin{array}{l}\text { at } 6 \text { month } \\
1 \text { year }\end{array}$ & 0 & 0 & 0 \\
\hline
\end{tabular}

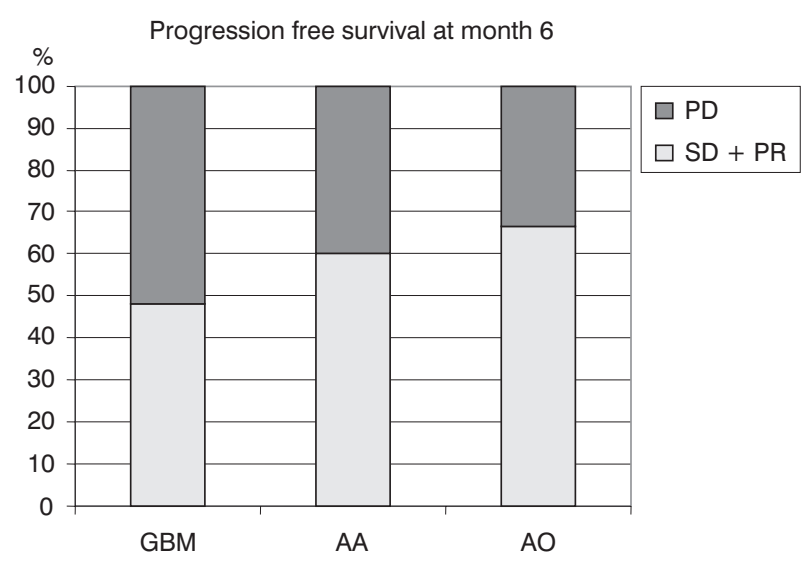

Fig. 1. Progression-free survival (sum of patients with partial response and stable disease) for all 37 patients treated with $\mathrm{POH}$ intranasal delivery. The median PFS rate was $48.2 \%$ for patients with GBM, $60 \%$ for patients with AA and $66.6 \%$ for patients with AO. Abbreviation: GBM - glioblastoma multiforme; AA anaplastic astrocytoma; AO - anaplastic oligodendroglioma; PD progressive disease; PR - partial response; $\mathrm{SD}$ - stable disease.

were analyzed by TUNEL assay. No apoptotic cells were detected in the sample from the first surgery. In contrast, cells positive for both TUNEL and caspase- 3 were detected in the sample following POH treatment (Fig. 4).

In the 5 patients with AA, 3 showed stable disease and 2 showed PD (Fig. 3 and Table 2). One patient with AO showed PR and remained alive without recurrence and no corticosteroids intake, 1 showed PD, and 1 showed stable disease (Fig. 3 and Table 2).

\section{Toxicity of $\mathrm{POH}$ intranasal administration}

All the patients were submitted to the toxicity tests required by National Cancer Institute Common Toxicity Criteria version 3 for this kind of clinical trial. Laboratory exams and images of the thorax, brain, and abdominal cavity were normal. Indeed, we now have 6 patients with more than one year of $\mathrm{POH}$. None of them presents any signs of toxicity. $\mathrm{POH}$ intranasal delivery was well tolerated in all patients. No dose reduction or drug discontinuation was required for any of the study participants.

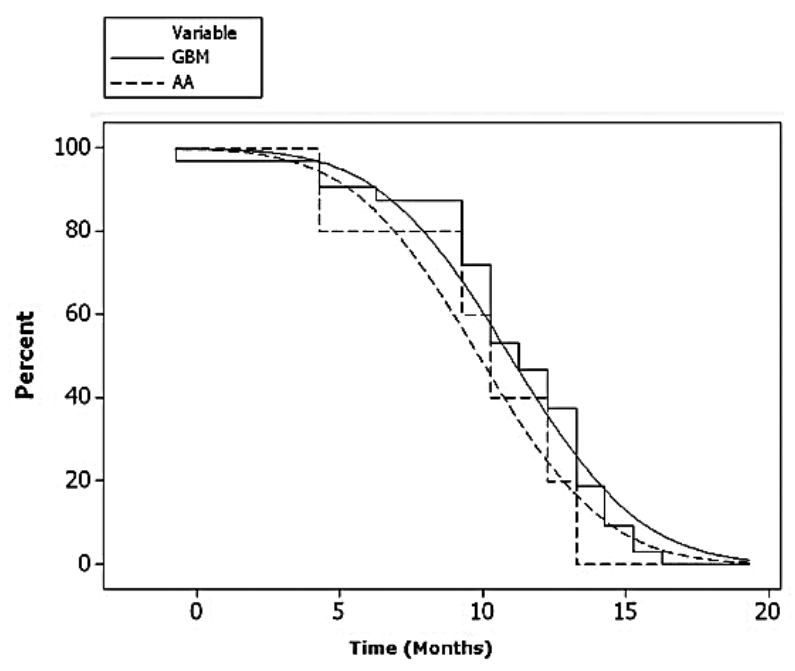

Fig. 2. Kaplan-Meier estimate of PFS for patients with GBM and AA.

\section{DISCUSSION}

Over the past three decades we have made great strides in the treatment of most, but not all, brain tumors. Dramatic advances have occurred in diagnostic imaging, neurosurgery, neuroanesthesia, radiotherapy, and chemotherapy for CNS tumors. Unfortunately, our progress has not yet met our expectations. Because of the infiltrative nature of most primary brain tumors, neurosurgery can never be expected to be curative for the majority of gliomas. Because infiltrative tumors interdigitate with normal brain cells and are not highly sensitive to irradiation, one cannot expect radiotherapy to be curative without serious damage to normal brain cells. The hope for a cure, then, rests with chemotherapy. The ability to treat most advanced malignancies with classical cytotoxic DNA-damaging agents is limited, with little curative potential and rare durable remissions [15]. This has led to emphasis on the development of new therapeutic agents with novel mechanism of action.

Cancer researchers now have a unified concept to guide their search for specific genetic abnormalities. The genes and proteins that participate in the conversion of normal into malignant cells are also involved in the key processes that convert extracellular events that culminate in division and growth [27]. Malignant gliomas commonly overexpress EGF receptor and its ligand-independent mutant EGFRvIII. This results in signaling through the Ras-MAPK and PI3K/Akt pathways [23]. Data showing that $\mathrm{POH}$ inhibits the prenylation of Ras and other proteins in many cell types [30] indicate that $\mathrm{POH}$ may be effective against glioma cells. In fact, our in vitro and in vivo experiments showed that $\mathrm{POH}$ induced marked changes in cell morphology, induced apoptosis, and led to inhibition of angiogenesis and migration of glioma cell lines and explanted cells 


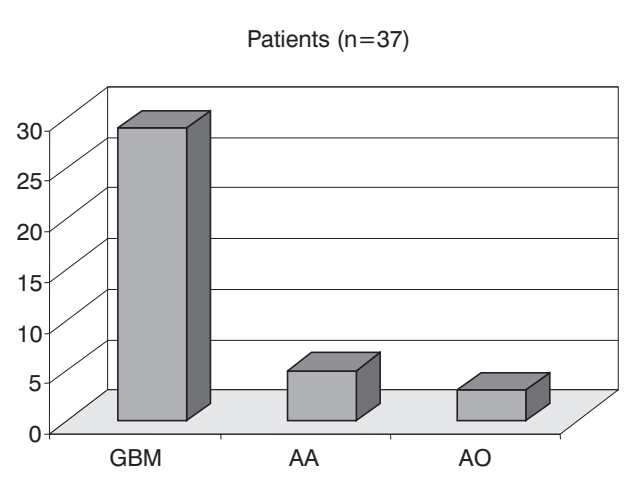

$\operatorname{GBM}(n=29)$

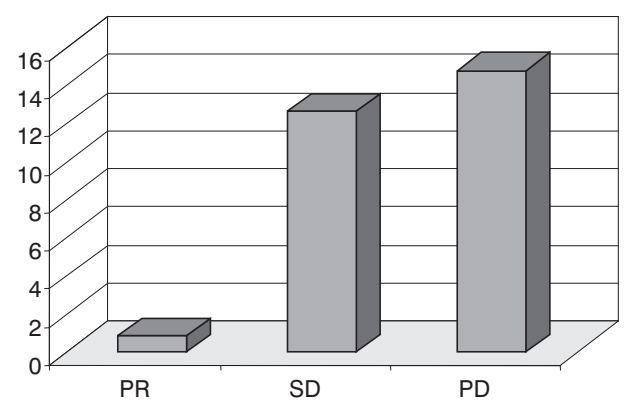

$\mathrm{AA}(\mathrm{n}=5)$

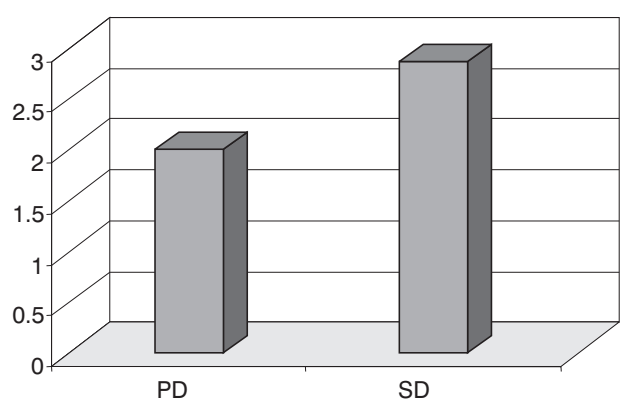

$\mathrm{AO}(\mathrm{n}=3)$

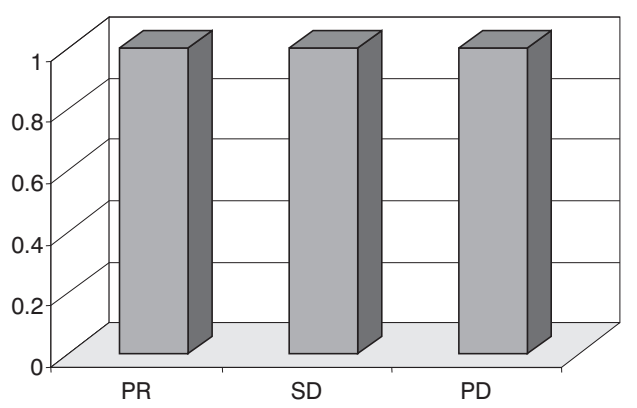

Fig. 3. Response evaluation of a total of 37 patients on the sixth month. Twenty-nine patients with GBM: 1 with PR, 13 with SD and 15 with PD; 5 patients with AA: 3 with SD and 2 with PD; 3 patients with AO: 1 with PR, 1 with SD and 1 with PD.
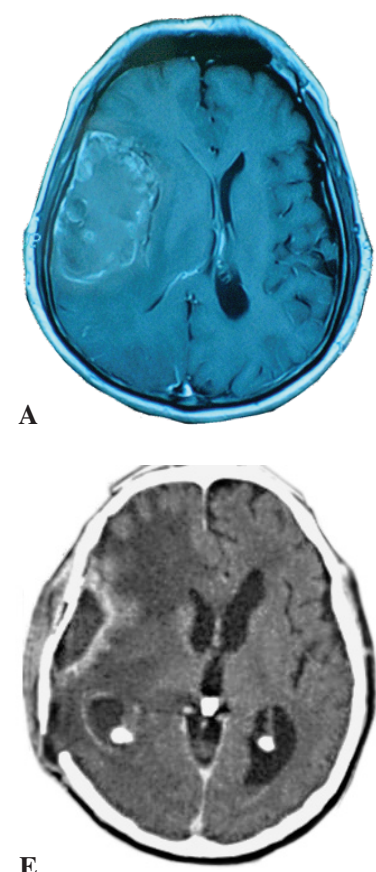
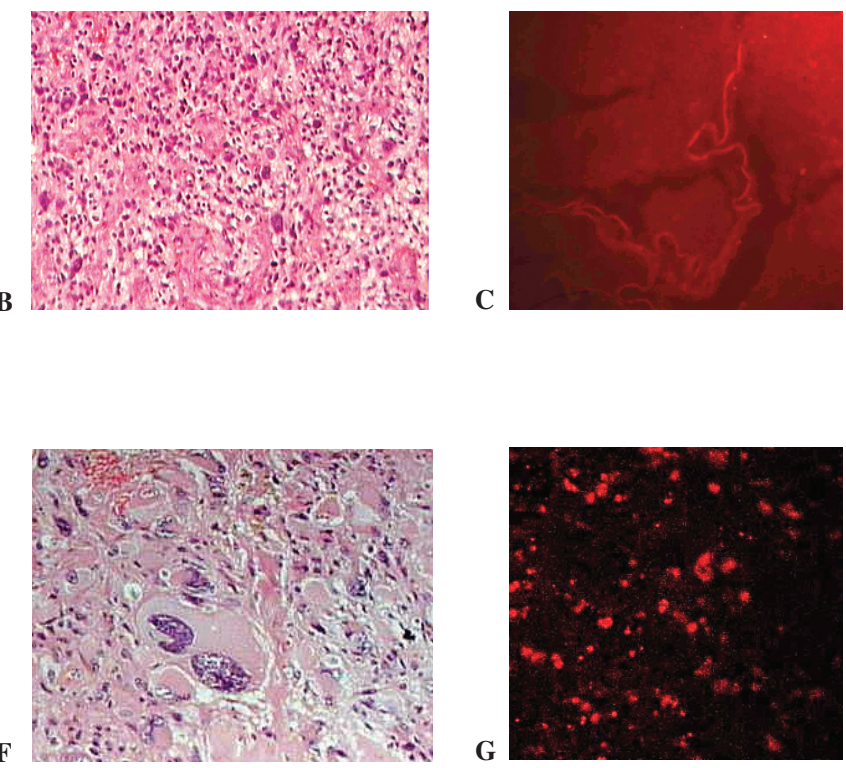
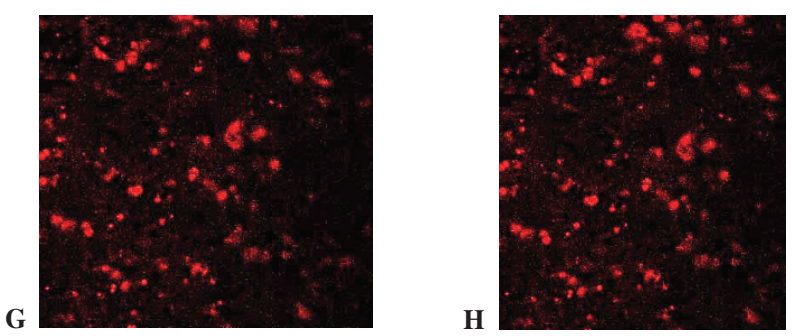

Fig. 4. Apoptotic responses in human recurrent glioblastoma multiforme treated with intranasal administration of POH. A: initial GBM; B: Astrocytic tumor of the highest grade with dense cellularity and striking pleomorphism (HE $\times 100)$; C, D: treated initial GBM by activated caspase-3 immunohistochemistry and terminal deoxynucleotidyl-mediated deoxyuridine triphosphate nick-end labeling (TUNEL). We found apoptotic cells in the treated tumor $\mathbf{G}$ : by activated caspase-3 immunohistochemistry and $\mathbf{H}$ : terminal deoxynucleotidyl-mediated deoxyuridine triphosphate nick-end labeling (TUNEL); E: computed tomography demonstrating a recurrent glioblastoma after radiation therapy and response to intranasal delivery of perillyl alcohol after 6 months treatment; F: scattered apoptotic cells (tumor cells) related to the areas with radiation and $\mathrm{POH}$ treatment effects $(\mathrm{HE} \times 400)$. 
from a patient with GBM [7, 12, 31]. Also, studies from our group indicate that $\mathrm{POH}$ may induce regression in patients with GBM $[8,9]$. Studies of phase I and II clinical trials with $\mathrm{POH}$ administered orally indicated that $\mathrm{POH}$ may have a tumoristatic effect and that the optimal schedule of administration would result from continuous drug exposure [2, 3]. However, these studies also showed toxicities, consisting of nausea, early satiety, eructation, and unpleasant taste as well as fatigue. The chronic nature of these toxicities led to problems with patient tolerance and compliance, suggesting that oral consumption of $\mathrm{POH}$ is an unpleasant experience for most patients. Our studies showed that the intranasal formulation of $\mathrm{POH}$ was generally well tolerated and relatively well absorbed. In some patients, this therapeutic strategy led to an increase in PFS and decrease in tumor size, as evidenced by MRI. It is interesting to note that in our study we investigated whether the partial regression observed in a patient with a recurrent GBM after treatment by intranasal delivery of $\mathrm{POH}$ four times daily, is mediated by apoptosis. Data from classical histology, terminal deoxynucleotidyl-mediated deoxyuridine triphosphate nick-end labeling (TUNEL) assay, as well as activation of caspase-3, showed increased apoptosis in the treated tumor (Fig. 4).

A study [24] showed that temozolomide schedules deplete O6-methylguanine methyltransferase and may overcome chemoresistance. This study enrolled 19 patients (15 with anaplastic AA, 4 with AO) who received temozolomide $\left(100 \mathrm{mg} / \mathrm{m}^{2} /\right.$ day for 21 consecutive days every 28-day cycle) at first recurrence, either until disease progression or for 12 cycles. Six-month PFS was $56 \%$, comparing favorably with historic controls treated with the standard 5-day temozolomide schedule. Median survival was 12.9 months (95\% confidence interval: 3.7-22 months). Among 15 evaluable patients, 2 had a complete or PR and 10 had stable disease. Grades 3 and 4 lymphopenia occurred in 53\% and $47 \%$ of the patients, respectively. However, treatment of recurrent malignant gliomas with intranasal delivery of POH achieved at six-month PFS was $48.2 \%$ for patients with GBM, $60 \%$ for patients with AA, and $66.6 \%$ for patients with AO. It is important to note that after 12 months of treatment with $\mathrm{POH}$ by intranasal delivery, 3 patients with malignant gliomas presented with considerable reductions in tumor mass and compression effects and 2 patients, 1 with GBM and 1 with AO, ceased corticosteroid intake. There were no toxicity events.

A common property of brain tumors is their ability to cause edema in the surrounding brain. Edema forms as a result of a leaky $\mathrm{BBB}$ and persists when the brain fails to clear the excess fluid. It is a significant source of morbidity and mortality. Although the experimental use of $\mathrm{POH}$ intranasal delivery in patients with refractory high-grade malignant gliomas has indeed led to some clinical responses, MRI offers evidence that there is no regression in edema peritumoral (Figs. 5 and 6).
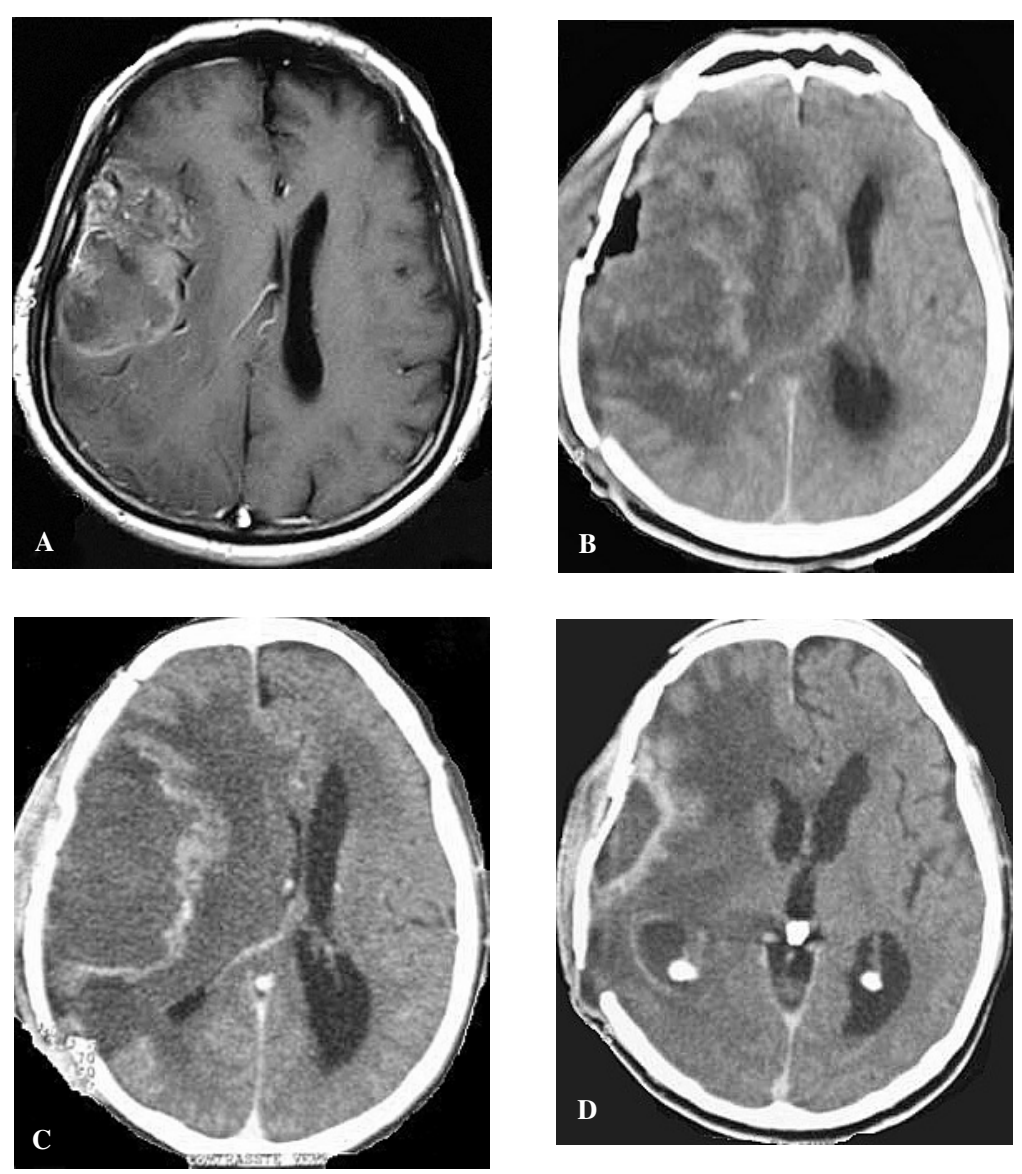

Fig. 5. Preoperative MRI revealing the extent of the tumor in the right hemisphere (A). Computed tomography demonstrating a recurrence of tumor after surgery and radiation therapy (B). Response to intranasal delivery of $\mathrm{POH}$ after 3 (C) and 6 months (D) treatment. We can observe persistent peritumoral edema. 

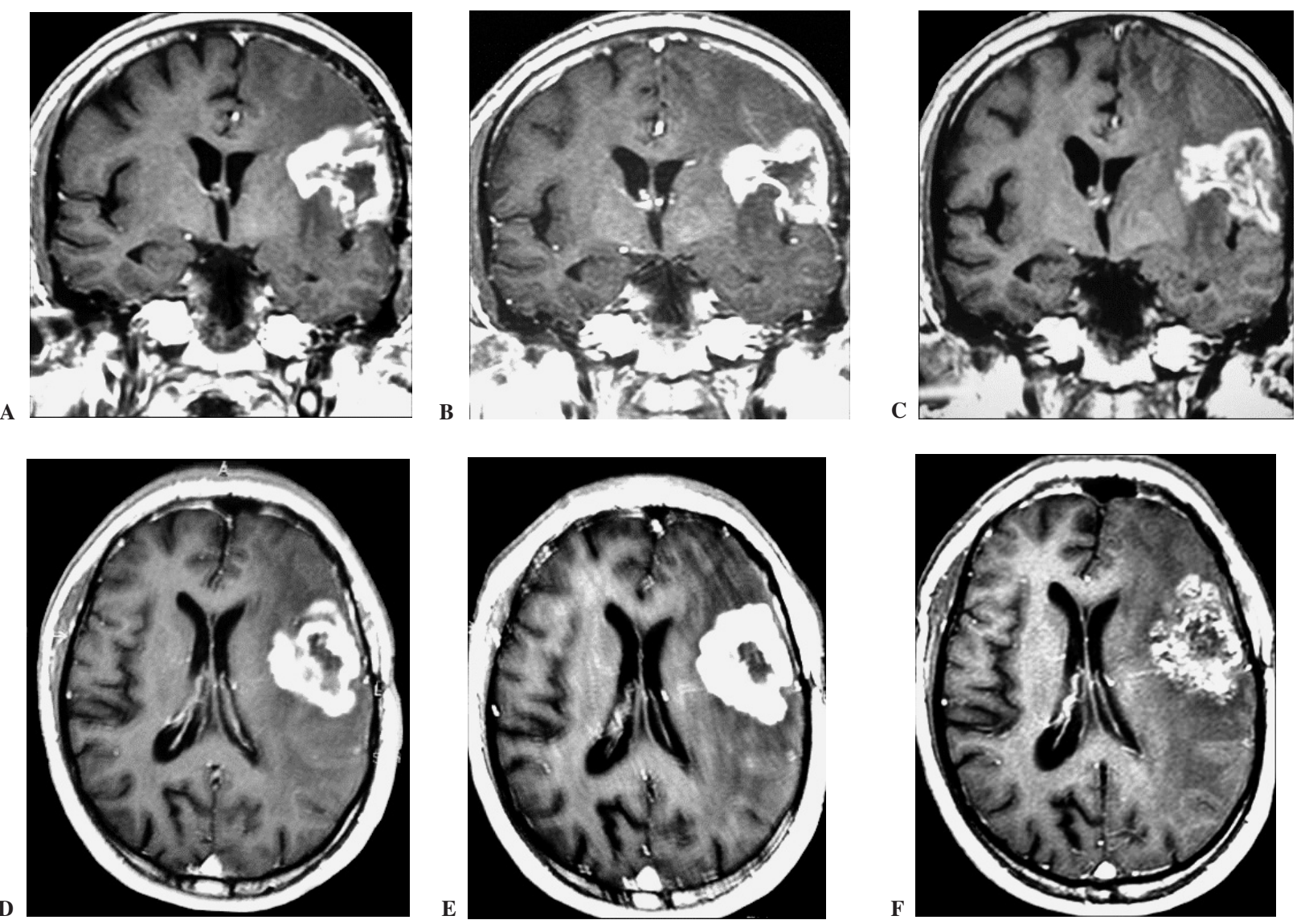

Fig. 6. Magnetic resonance imaging (MRI) showing the effect to intranasal delivery of POH treatment in a patient with relapsed glioblastoma. Note the persistent peritumoral edema between the initial MRI (A, D) and the follow-up performed after 6 months $(\mathbf{B}, \mathbf{E})$ and after 18 months $(\mathbf{C}, \mathbf{F})$ of treatment.

In summary, despite dramatic advances in the appreciation of the underlying molecular biology of malignant gliomas, we remain ignorant of many crucial aspects of glioma biology. Regulators of tumor invasion escape immune targeting and neo-angiogenesis remains to be elucidated. An invasive and hypoxic tumor shows resistance to traditional therapies and, probably, targeted therapies. It is increasingly evident that a greater understanding of the molecular mechanisms of gliomagenesis is needed to improve treatments for these patients. Rather than being paralyzed by these intricacies, the neuro-oncology community, pharmaceutical companies, and governmental regulatory agencies will need to attack these issues head-on. Success in changing the outcome of malignant glioma depends on refining our understanding of the biology and biochemistry of brain tumors, enabling us to develop new therapeutic opportunities against this disease.

In view of the fact that the proliferative signaling of human high-grade gliomas seems to utilize p21-Ras-mediated signal transduction, the concept of using p21-Ras pathway inhibition, whether by prenylation blockade or by other pharmacological approaches, as an adjuvant or single therapy appears to be attractive. $\mathrm{POH}$ intranasal administration is safe, noninvasive, and low-cost and regression of tumor size in some patients is suggestive of antitumor activity. However, it should also be taken into account that human tumors often contain multiple oncogenic mutations at the time of diagnosis. These may occur in upstream signaling elements such as the EGF receptor and Ras and/or in downstream elements such as Raf-1, the MAPK cascade. In addition, neoplastic cells can activate various alternative signaling routes upon blockage of Ras. Therefore it may be too much to expect that Ras therapies alone can be successful against high-grade malignant gliomas in the clinic. Perhaps such therapies may be studied in combination with other treatment modalities such as VEGF, mTOR, PDGFR, and EGFR inhibitors. In this regard the interaction of neoplastic cells with the peritumoral stroma can also result in the disruption of tissue homeostasis. Tumor burden frequently results in the recruitment of leukocytes to the tumor site (through cytokines and chemokines), which leads to an inflammatory reaction. Such approaches may help further refine our understanding of the biology and biochemistry of brain tumors, enabling us to develop new therapeutic opportunities against this disease. 


\section{REFERENCES}

1. Bahl J. J., De Armond R. L. and Bressler R. (2004): Multisite inhibition by phenylacetate of PC-3 cell growth. Anticancer Drugs, 15, 513-523.

2. Bailey H. H., Attia S., Love R. R., Fass T., Chappell R., Tutsch K., Harris L., Jumonville A., Hansen R., Shapiro G. R. and Stewart J. A. (2008): Phase II trial of daily oral perillyl alcohol (NSC 641066) in treatment-refractory metastatic breast cancer. Cancer Chemother. Pharmacol., 62, 149-157.

3. Bailey H. H., Wilding G., Tutsch K. D., Arzzomanian R. Z., Albert D., Feierabend C., Simon K., Marnocha R., Holstein S. A., Stewart J., Lewis K. and Hohl R. J. (2004): A phase I trial of perillyl alcohol administered four times daily for 14 days out of 28 days. Cancer Chemother. Pharmacol., 54, 368-376.

4. Brat D. J., Scheithauer B. W., Fuller G. N. and Tihan T. (2007): Newly Codified Glial Neoplasms of the 2007 WHO Classification of Tumours of the Central Nervous System: Angiocentric Glioma, Pilomyxoid strocytoma and Pituicytoma. Symposium: The 2007 Revised World Health Organization (WHO) Classification of Tumours of the Central Nervous System: Newly Codified Entities.

5. Buechler R. D. and Peffley D. M. (2004): Proto oncogene/eukaryotic translation initiation factor (eIF) $4 \mathrm{E}$ attenuates mevalonate-mediated regulation of 3-hydroxy-3-methylglutaryl coenzyme A (HMG-CoA) reductase synthesis. Mol. Carcinog., 41, 39-53.

6. Campbell P. M., Groehler A. L., Lee K. M., Ouellette M. M., Khazak V. and Der C. J. (2007): K-Ras promotes growth transformation and invasion of immortalized human pancreatic cells by Raf and phosphatidylinositol 3-kinase signaling. Cancer Res., 67, 2098-2106.

7. Da Fonseca C. O. (2004): Effects of in vivo and in vitro treatment of monoterpene perillyl alcohol on proliferation and gene expression control of high grade gliomas. Arq. Neuro-Psiquiatr., 62, 1117-1118.

8. Da Fonseca C. O., Landeiro J. A., Clark S. S., QuiricoSantos T., Da Costa Carvalho M. G. and Gattass C. R. (2006): Recent advances in the molecular genetics of malignant gliomas disclose targets for antitumor agent perillyl alcohol. Surg. Neurol., 65 (suppl. 1), S2-8.

9. Da Fonseca C. O., Masini M., Futuro D., Caetano R., Gattass R. C. and Quirico-Santos T. (2006): Anaplastic oligodendroglioma responding favorably to intranasal delivery of perillyl alcohol: a case report and literature review. Surg. Neurol., 66: 611-615.

10. Duncan R. E., Lau D., El-Sohemy A. and Archer M. C. (2004): Geraniol and beta-ionone inhibit proliferation, cell cycle progression, and cyclin-dependent kinase 2 activity in MCF-7 breast cancer cells independent of effects on HMG-CoA reductase activity. Biochem. Pharmacol., 68, 1739-1747.

11. Feldkamp M. M., Lau N., Roncari L. and Guha A. (2001): Isotype-specific Ras.GTP-levels predict the efficacy of farnesyl transferase inhibitors against human astrocytomas regardless of Ras mutational status. Cancer Res., 61, 4425-4431.

12. Fernandes J., Fonseca C.O., Teixeira A. and Gatass C. R. (2005): Perillyl alcohol induces apoptosis in human glioblastoma multiforme cells. Oncol. Rep., 13, 943-947.

13. Franco-Hernández C., Martínez-Glez V. and Rey J. A. (2007): Biology molecular of glioblastomas. Neurocirugia, 18, 373-382.
14. Guha A., Feldkamp M. M., Lau N., Boss G. and Pawson A. (1997): Proliferation of human malignant astrocytomas is dependent on Ras activation. Oncogene, 15, 2755-2765.

15. Hofer S. and Herrmann R. (2001): Chemotherapy for malignant brain tumors of astrocytic and oligodendroglial lineage. J. Cancer Res. Clin. Oncol., 127, 91-95.

16. Holstein S. A. and Hohl R. J. (2003): Monoterpene regulation of Ras and Ras-related protein expression. J. Lipid Res., 44, 1209-1215.

17. Huanbiao M. O. and Elson C. E. (2004): Studies of the isoprenoid-mediated inhibition of mevalonate synthesis applied to cancer chemotherapy and chemoprevention. Exp. Biol. Med., 229, 567-585.

18. Inda M. M., Fan X., Muñoz J., Perot C., Fauvet D., Danglot G., Palacio A., Madero P., Zazpe I., Portillo E., Túnón T., Martínez-Peńuela J.M., Alfaro J., Eiras J., Bernheim A. and Castresana J. S. (2003): Chromosomal abnormalities in human glioblastomas: gain in chromosome $7 \mathrm{p}$ correlating with loss in chromosome 10q. Mol. Carcinog., 36, 6-14.

19. Jeuken J. W., Sprenger S. H., Vermeer H., Kappelle A. C., Boerman R. H. and Wesseling P. (2002): Chromosomal imbalances in primary oligodendroglial tumors and their recurrences: clues about malignant progression detected using comparative genomic hybridization. J. Neurosurg., 96, 559-564.

20. Jeuken J., van den Broecke C., Gijsen S., Boots-Sprenger S. and Wesseling P. (2007): RAS/RAF pathway activation in gliomas: the result of copy number gains rather than activating mutations. Acta Neuropathol., 114, 121-133.

21. Karp J. E. and Lancet J. E. (2007): Development of farnesyltransferase inhibitors for clinical cancer therapy: focus on hematologic malignancies. Cancer Invest., 25:484-494.

22. Konstantinopoulos P. A., Karamouzis M. V. and Papavassiliou A. G. (2007): Post-translational modifications and regulation of the RAS superfamily of GTPases as anticancer targets. Nat. Rev. Drug Discov., 6, 541-555.

23. Mischel P. S. and Cloughesy T. F. (2003): Targeted molecular therapy of GBM. Brain Pathol., 13, 52-61.

24. Neyns B., Chaskis C., Joosens E., Menten J., D'Hondt L., Branle F., Sadones J., Michotte A. (2208): A multicenter cohort study of dose-dense temozolomide (21 of 28 days) for the treatment of recurrent anaplastic astrocytoma or oligoastrocytoma. Cancer Invest., 26, 269-277.

25. Nigro J. M., Misra A., Zhang L., Smirnov I., Colman H., Griffin C., Ozburn N., Chen M., Pan E., Koul D., Yung W. K., Feuerstein B. G. and Aldape K. D. (2005): Integrated array-comparative genomic hybridization and expression array profiles identify clinically relevant molecular subtypes of glioblastoma. Cancer Res., 65, 1678-1686.

26. Omerovic J., Laude A. J. and Prior I. A. (2007): Ras proteins: paradigms for compartmentalised and isoform-specific signalling. Cell. Mol. Life. Sci., 64, 2575-2589.

27. Rinker-Schaeffer C. W., O'Keefe J. P., Welch D. R. and Theodorescu D. (2006): Metastasis suppressor proteins: discovery, molecular mechanisms, and clinical application. Clin. Cancer Res., 12, 3882-3889.

28. Ruocco A., Santillo M., Cicale M., Serù R., Cuda G., Anrather J., Iadecola C., Postiglione A., Avvedimento E. V. and Paternò R. (2007): Farnesyl transferase inhibitors induce neuroprotection by inhibiting Ha-Ras signalling pathway. Eur. J. Neurosci., 26, 3261-3266.

29. Salazar M. D. (2004): Effects of monoterpene perillyl alcohol intranasal administration in peripheral lymphoid population and mucosal immune system of $\mathrm{C} 57 \mathrm{BL} / 6$ mice. 
Laboratório de Patologia Celular, Instituto de Biologia, Universidade Federal Fluminense, Brazil.

30. Stayrook K. R., McKinzie J. H., Barbhaiya L. H. and Crowell P. L. (1998): Effects of the antitumor agent perillyl alcohol on H-Ras vs. K-Ras farnesylation and signal transduction in pancreatic cells. Anticancer Res., 18, 823-828.
31. Teruszkin I., Alves S., Silva H. N., Curie C. M., Bozza M., Fonseca C. O. and Da Costa C. M. (2002). Effects of perillyl alcohol in glial cell line in vitro and anti-metastatic activity in chorioallantoic membrane model. Int. J. Mol. Med., 10, 785-788. 